\title{
SMALL VOLUNTARY MOVEMENTS OF THE EYE*
}

\author{
BY \\ B. L. GINSBORG \\ Physics Department, University of Reading
}

IT is well known that the transfer of the gaze from one point to another, where the points are separated by large angles, is not accomplished in a single smooth movement. An investigation by Lord and Wright (1949) of movements of angles of $5^{\circ}$ and less has shown that the same is true of smaller angles. In the present paper a detailed investigation of the small eye movements which occur when the gaze is transferred from one fixation point to another is described.

The work arose out of and employed the same method as an investigation of the small involuntary movements which occur when the subject keeps his gaze as steady as possible (Ditchburn and Ginsborg, 1953). The experiments were carried out on two subjects (B.L.G. and D.M.M.) and were concerned with angular movements from 20-min. arc to $4^{\circ}$. Results are reported for the time sequence and angular pattern of the eye movements and for the reaction time of the eye.

\section{Methods}

The subjects were seated in a medical examination chair which provided support for the head and neck. The head was immobilized by biting on a rigidly mounted dental impression mouthpiece. The fixation targets were $450 \mathrm{~cm}$. from the subjects' eyes and consisted of bright spots (about 0.02 c.p.) each subtending 2-min. arc at the eye.

The subjects wore contact lenses on which small plane mirrors had been worked (Ginsborg and Heavens, 1951). A fixed incident beam was directed on to each mirror and the reflected beam was directed on to continuously moving photographic paper. When the contact lens rotated through a small angle, the reflected beam was rotated through twice that angle. A continuous record of the rotations of the contact lenses could therefore be obtained. Provided there is no relative movement between the contact lens and the eye, this record then allows the movements of the eye to be deduced.

The optical system used in the first series of experiments (Method A) is shown diagrammatically in Fig. 1 (opposite).

$\mathrm{I}$ is the source of illumination (a high-pressure mercury arc) which is focused by the lens $\mathrm{L}_{1}$ on to a pinhole aperture 0 . The light is them made parallel by the lens $\mathrm{L}_{2}$ and illuminates two slits, $S_{1}$ which is vertical and $S_{2}$ which is horizontal. The lens $L_{3}$ focuses the pinhole 0 on to the mirror on the contact lens, and also focuses the slits $S_{1}$ and $S_{2}$ on to the photographic paper $P$. The cylindrical lens $L_{1}$ and the horizontal slit $S_{3}$ serve to provide a high intensity image of the edges of the slits on the paper. V.A. is the direction of the visual axis.

Two separate traces are produced on the photographic paper in the following manner:

When the mirror rotates about a vertical axis, $S_{2}$ (the image of $S_{2}$ ) is deflected to the left or to the right and thus causes a deflection of the trace. When the mirror rotates about the horizontal axis, $S_{2}$ moves upwards or downwards along its own length causing no deflection of the trace.

*Received for publication October 23, 1952. 


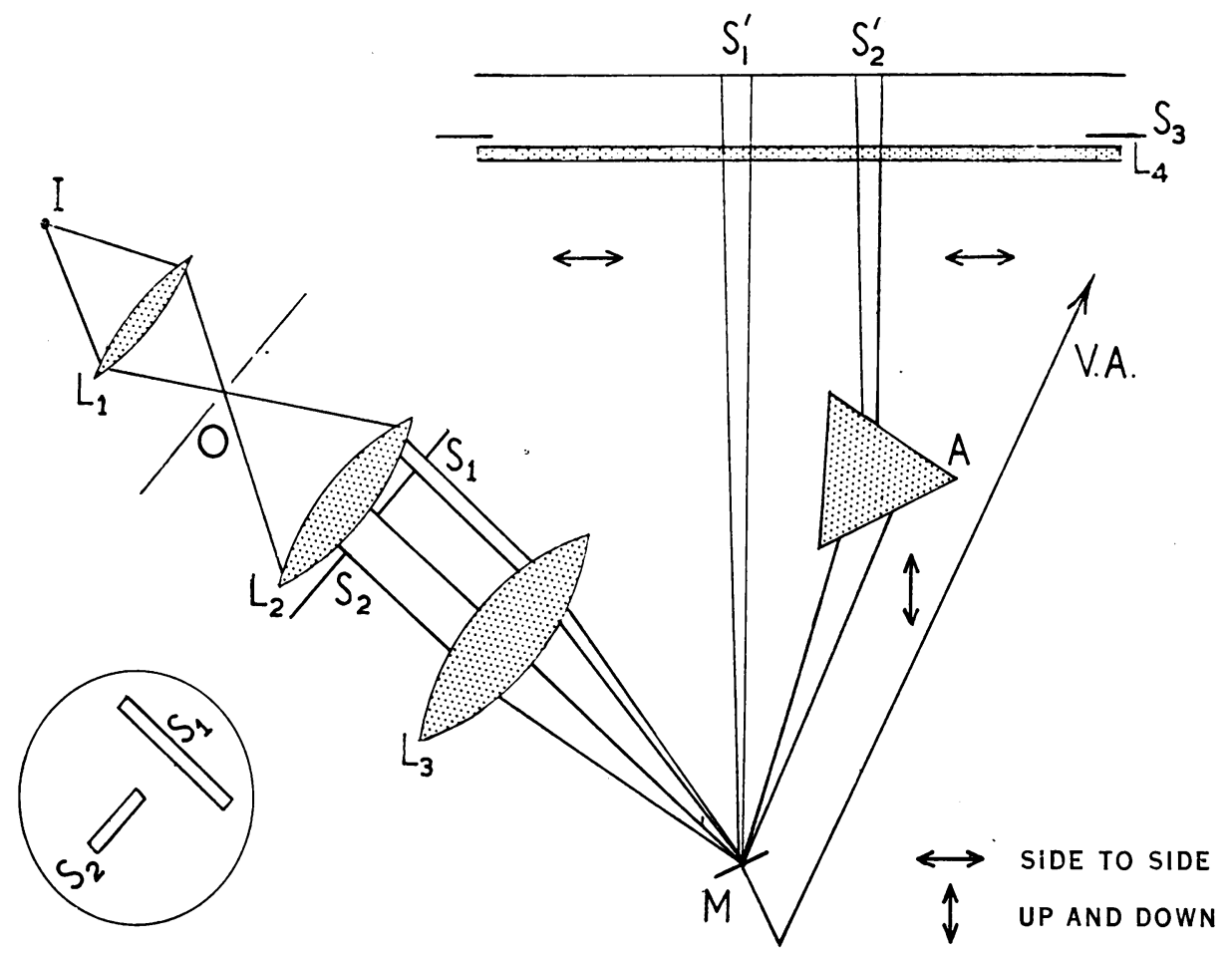

FIG. 1.-Optical system used in all experiments.

The vertical slit $\mathbf{S r}$ therefore serves to record the component about the vertical axis of the rotation of the eye. The image of the horizontal slit $S^{\prime}{ }_{1}$ moves upwards and downwards when the mirror rotates about a horizontal axis and moves along its own length when the mirror rotates about a vertical axis. The upwards and downwards movements of the image, which are proportional to the rotation of the eye about a horizontal axis, are converted into a sideways deflection of the trace by interposing the right-angled prism $\mathrm{A}$, with its base tilted $45^{\circ}$ to the horizontal, in the beam.

The smallest rotation which could be detected was about 12-sec. arc. The duration of any movement could be measured to about 5 millisec.

In a second series of experiments (Method B) the optical system was modified. The slits were replaced by a pinhole aperture, the image of which was projected on to a stationary plate which replaced the continuously moving photographic paper. The image of the pinhole moved directly with the eye so that the trace obtained was a projection of the eye movements on a frontal plane. The method has the obvious advantage that the angular pattern of the eye movements may be deduced immediately from the trace, whereas Method A requires a graphical construction. Method B is not however suitable for binocular recording nor is it possible to deduce the duration of a movement from the records. Method A was therefore used for experiments in which time relations or binocular records were required and Method B, for investigations of the angular patterns of eye movements.

\section{Results}

I. Movement of the Contact Lens relative to the Eye.-The possibility of relative movement between the eye and contact lens when the subject voluntarily transferred his gaze from a point $A$ to a second point $B$ was first investigated. In the case of involuntary movements of the eye (i.e. those which occur whilst the subject attempts to keep his gaze fixed), there is con- 
siderable indirect evidence (Ditchburn and Ginsborg, 1953) that the contact lens moves precisely with the eye. It is nevertheless clearly desirable to obtain direct evidence by comparing the rotation of the contact lens, as deduced from the records, with the known angular separation of the fixation targets A and B. The procedure adopted was as follows:

The subject fixated on a point $A$ for 10 sec. and a continuous record was taken for this period using Method A. The camera was then stopped and restarted at a signal from the subject that he was fixating on point $B$. The angular separation of A and B varied between 20 -min. arc and a $4^{\circ}$ arc. In some experiments the subject removed his head from the dental impression mouthpiece and relaxed between recordings of fixation on $\mathrm{A}$ and $\mathrm{B}$.

In all cases the mean separation of the traces agreed with the angular separation of the fixation points to \pm 3 -min. arc. During fixation the eye moves \pm 10 -min. arc around a central point. The above results thus provide confirmation that there is no relative movement between contact lens and eye, at least up to an angle of $4^{\circ}$.

IIA. Time Sequence of Small Eye Movements.-In order to investigate the time sequence of small eye movements, continuous records (Method A) were taken for periods of about $10 \mathrm{sec}$ :

The subject fixated on point $\mathrm{A}$ for 5 sec., rapidly transferred his gaze to point $\mathrm{B}$, and fixated on the latter for a further $5 \mathrm{sec}$. A selector switch, controlled either by the subject or an assistant, extinguished point A and illuminated point B simultaneously. The angular separation of $A$ and $B$ was again varied between 20 -min. arc and $4^{\circ}$ arc.

The general behaviour of the eye for angles between $1^{\circ}-4^{\circ}$, as deduced from records of the components of the movements, is shown in Fig. $2 a, b, c$ (opposite). The movement starts as a sharp saccade carrying the eye through an angle which varies from half the angular separation of A and B to an angle slightly greater than the angular separation between A and B. So far as can be seen from these records, the saccade occurs in three stages:

There is a period of acceleration to a maximum speed, this speed is maintained for a further period and finally there is a period of deceleration.

The mean velocity of rotation during the saccade varies with the excursion involved, increasing with larger excursions (Fig. 3, opposite). A more detailed investigation could be made by means of a high-speed drum camera.

The direction of the movement is not confined to the direction of separation of A and B. In those cases where a marked undershoot has occurred (and on the rare occasions where there is an overshoot) the saccade is followed by a period during which the eye moves comparatively little. Such movement as does occur during this period ranges in speed between $5 \mathrm{~min}$. arc per sec. and $2^{\circ}$ per sec. and may be either towards or away from the final fixation target. The minimum period of this drifting movement is $0.15 \mathrm{sec}$., and the maximum $0.88 \mathrm{sec}$. The correlation between duration of drift and "error" from the final fixation point is not very high, but large errors are invariably associated with short periods $(0 \cdot 15$ to $0 \cdot 30 \mathrm{sec}$.). Where a marked undershoot 


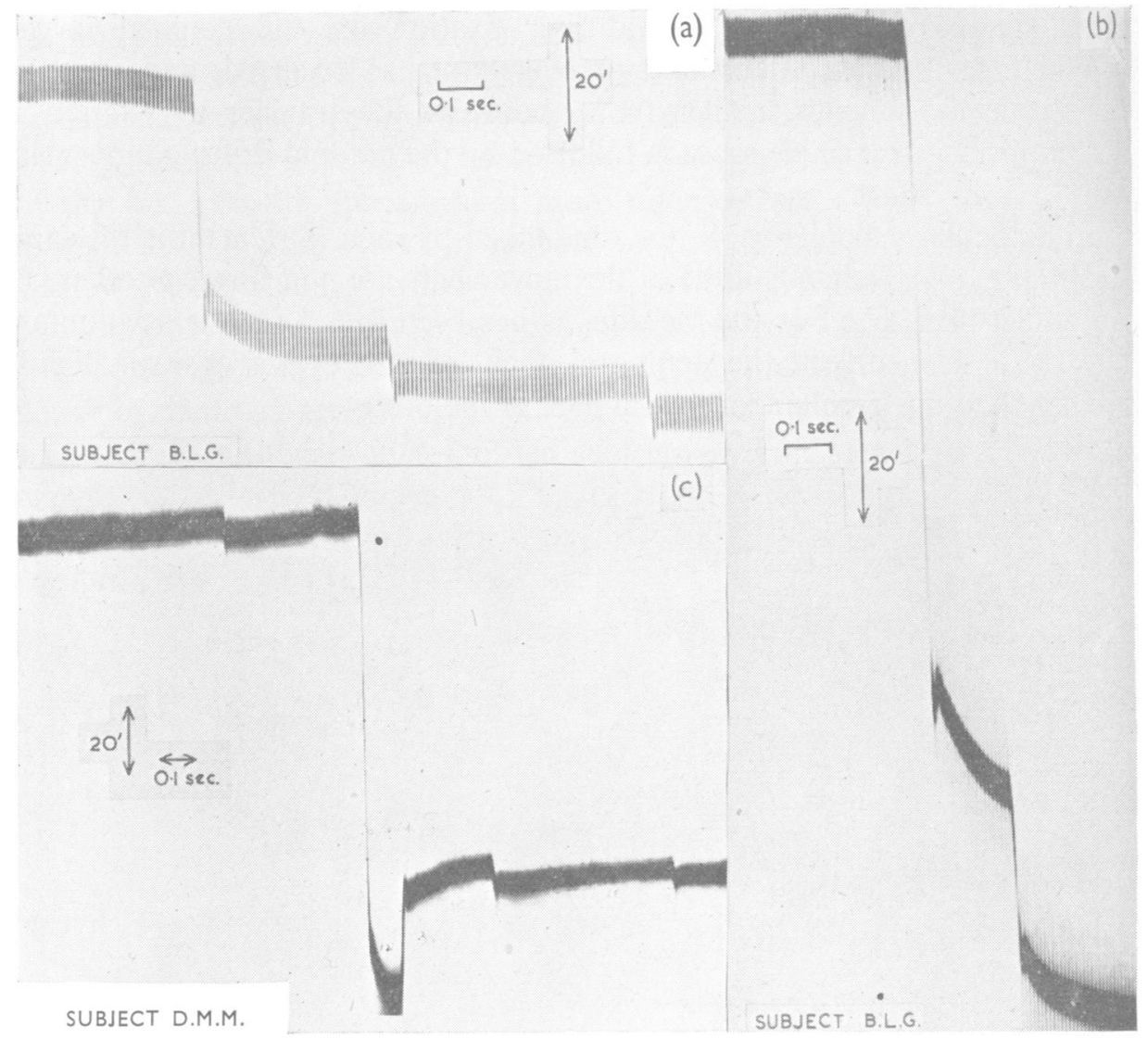

FIG. 2.-Component of rotation of the eye about a vertical axis when the gaze is transferred from one point to another. The points are on a horizontal line separated by the following angles: (a) $80 \mathrm{~min}$. arc. (b) $4^{\circ}$. (c) $2^{\circ}$.

Time increases towards the right.

or overshoot has occurred, the next stage of the movement is a further saccade which carries the eye to within about $10 \mathrm{~min}$. arc of the correct direction of fixation. This is followed by a gradual drift towards the fixation point. Normal involuntary eye movements corresponding to fixation are superimposed on this drift which may last up to one second.

The frequency of involuntary flicking during this drift and a further 2 or 3 seconds is, how-

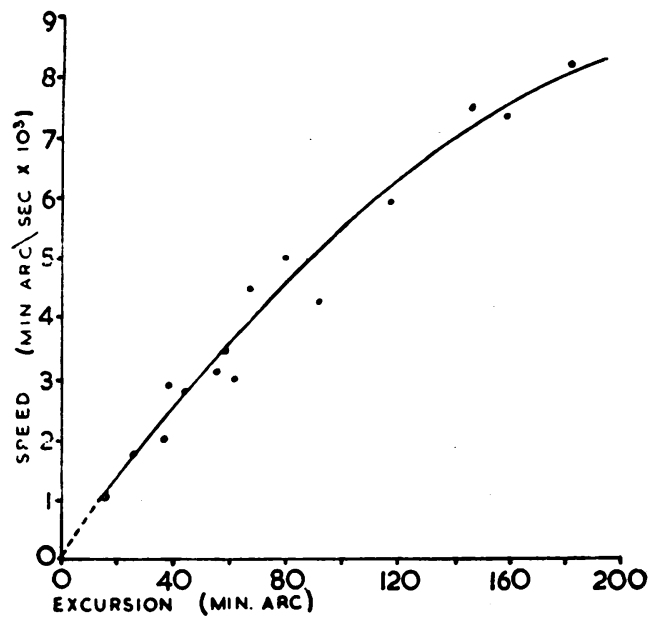

FIG. 3.-Relation between maximum speed occurring in saccade and magnitude of saccade. 
ever, significantly higher than during fixation, on the original target.

Where no marked undershoot or overshoot has occurred, and, in most cases, for movements smaller than about $1^{\circ}$, the transfer of fixation is accomplished by a single saccade followed by the gradual drifting movement as described above.

In binocular vision (Fig. 4), the time taken by each movement is the same for both eyes. The magnitude of the movements are not, however, identical for subject B.L.G. For the saccade, as has been found for the involuntary flick with this subject, the temporal flick of one eye is always slightly greater than the simultaneous nasal flick of the other.

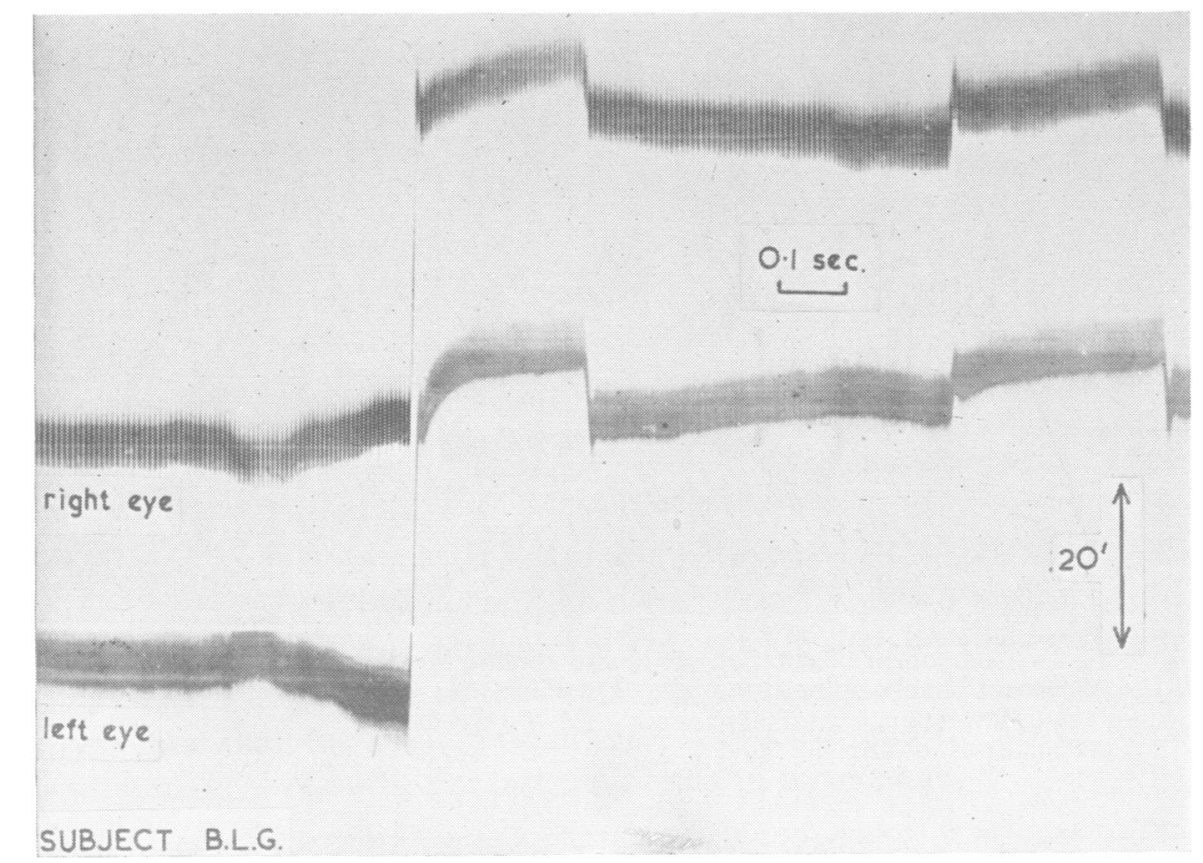

FIG. 4.-Gaze transferred through angle of $40 \mathrm{~min}$. arc. Components of rotation of both eyes about a vertical axis. Time increases towards the right.

IIB. Angular Pattern of Small Movements.-The angular pattern B of the movements may be deduced by combining the horizontal and vertical components of the rotation graphically. Method B, however, provides direct traces of the movements projected in a plane, and some idea of the relative speeds of the different types of movement is given by the intensity of the photographic trace. Fig. 5(a) illustrates the type of record obtained when the subject fixates successively on three points (A, B, and C) at the apices of a right-angled triangle. In this case the subject momentarily exposed the recording beam, using the hand as a shutter, when he believed he had achieved satisfactory fixation on each point in turn.

Figs $5(b)$ and $5(c)$ illustrate records taken when the subject fixated on each of three points at the apices of a triangle, transferring his gaze as rapidly 
as possible between each fixation, but maintaining his gaze on each point until he was satisfied he was fixating correctly. It can be seen that the first saccade carries the eye in a straight path, which, however, is not in a line joining the fixation points. The drifting movement which occurs at the end of the first saccade appears as a more intense trace. In upward oblique movements this drift occurs in a characteristically curved path. Figs $5(d)$ and $5(e)$ were obtained when the gaze was transferred between two points on a horizontal line, and two points on a vertical line. It is clear that in this type of experiment, where the eye moves from point to point, the subject does not fixate as accurately as is possible during prolonged fixation on a single target, although he is satisfied subjectively that he has transferred his gaze to the new fixation point. This observation is confirmed by the continuous component records described above.

(a)

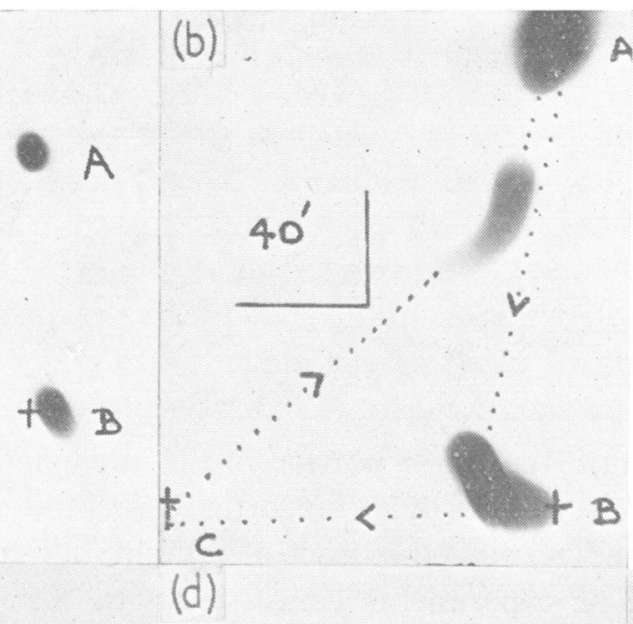

Fig. 5.-Projection of rotation of left eye on a stationary plate. The rapid saccadic movements visible in the original records are shown by dotted lines. The unbroken lines represent displacements of 40 min. arc in horizontal and vertical directions. The crosses show the positions of the fixation points $\mathrm{B}$ and $\mathrm{C}$ relative to $\mathrm{A}$.

(c)

(d)

(e)

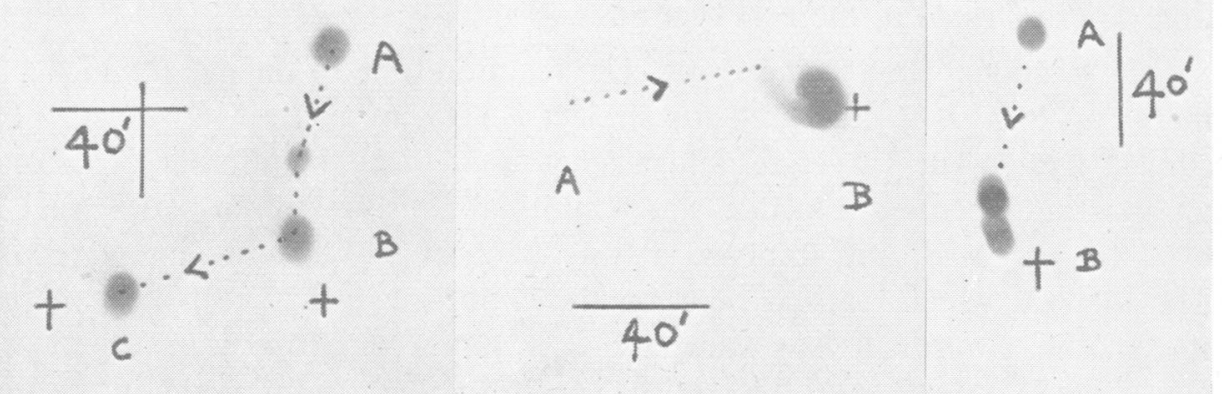

III. "Accuracy" of Direction of Fixation after Larger Eye Movements.A comparison of the experiments described in Section I with those in Sections IIA and IIB shows that if the subject is allowed an indefinite time to fix his gaze on a target, the image falls on a small and definite area of the fovea. Nevertheless, subjectively satisfactory fixation is obtained, although the image falls outside this region. In order to extend observations of this phenomenon, the following series of experiments were carried out using Method A. 
The subject fixated on a point separated from the final fixation point by an angle of $20^{\circ}-30^{\circ}$. The initial fixation point was then extinguished and the subject kept his gaze as steady as possible, in total darkness. After a short period, the final fixation point was illuminated together with a time mark on the record. In order to maintain the sensitivity of recording only the terminal portion of the movement was recorded.

The angular separation between the visual axis, when the subject first considered fixation satisfactory and final direction of fixation was about $1^{\circ}$. After this time the subject was, on most occasions, not conscious that further eye movement was occurring. Nevertheless a gradual drift did take place, until finally the mean position of the visual axis was within \pm 3 min. arc of the calculated direction for fixation on the final target. The times taken for the movements in one series of experiments are given in the Table.

TABLE

TIME BETWEEN ILLUMINATION OF TARGET AND VISUAL AXIS TO REACH SEPARATION

(i) FROM TARGET OF $1^{\circ}$ ARC

(ii) FROM TARGET OF \pm 3 MIN. ARC. (Total movement $20^{\circ}$ )

\begin{tabular}{c|c|c}
\hline Target & $1^{\circ}$ arc & \pm 3 min. arc. \\
\hline & 0.28 & 1.91 \\
Time & 0.16 & 1.65 \\
(sec.) & 0.29 & 2.23 \\
& 0.32 & 1.68 \\
& 0.43 & 3.12 \\
\hline
\end{tabular}

The times taken for the major portion of the movement cannot be deduced from those quoted in the Table, since these include the reaction time of the eye, i.e. the time between the onset of illumination and the beginning of the movement. The experiments concerned with the measurement of this time are described in the next section.

IV. Reaction Time of the Eye.-The reaction time of the eye for measurements up to $4^{\circ}$ was measured for subject B.L.G. by using Method A. The subject fixated on Target $A$, and after a small random number of seconds an assistant operated a switch which simultaneously extinguished Target A, exposed Target $\mathrm{B}$, and imposed a time trace on the eye-movement record. The interval which elapsed between the exposure of Target $B$ and the eye movement towards it was variable. On some occasions the subject was conscious of a delay, and the records confirmed that an interval considerably larger than the minimum reaction time had elapsed. This minimum time was $0 \cdot 14 \mathrm{sec}$, a period almost identical with the minimum time between two saccades when a voluntary transfer of the gaze occurs in more than one stage. In some of the records a small non-saccadic movement of the eye was observed about 60 msec. after the exposure of the second target. This movement was less marked or absent when the time marker was not used. Both the selector switch and the time marker were not completely silent, the time marker being noisier than the selector switch alone. It therefore seems possible that the non-saccadic movement is a response to the auditory signal involved in the change of target. 


\section{Discussion}

The recording of small eye movements by sensitive methods has been directed almost entirely to the study of their effects on visual perception. The present paper for example reports part of a larger investigation with a twofold aim:

(a) to examine the involuntary movements of the eye which occur when a subject attempts to keep his gaze fixed.

(b) to study the effect of eliminating movements of the visual image relative to the retina.

The method of investigation involved the use of a contact lens, and clearly the validity of the results which were obtained depends in the first place on the precision with which the contact lens moved together with the subject's eye. The results reported here offer no evidence to suggest that the contact lens moves independently of the eye. In fact, the experiments reported in Section I constitute positive evidence that the lens moves with the eye with a high degree of precision.

The records of involuntary movements during fixation show that the visual image can be maintained on a fixed region of the fovea about $100 \mu$ in diameter. When the gaze is transferred to a second point, the new visual image eventually falls on and is maintained on this same region of the fovea. It might therefore be thought that subjectively adequate fixation occurs only when the image falls on this region. The experiments described in Section II show that this is not the case, which suggests that two separate processes are involved in the movement of the visual axis towards a fixation point. The first process is concerned with establishing the visual image within the central $1^{\circ}$ region of the fovea. This is achieved by one or more saccades, the interval between saccades never being less than the minimum reaction time of the eye. The second of two saccades is therefore comparable to the reflex response to the exposure of a new visual stimulus. Shortly after the end of the first process, the subject has a sense of adequate fixation. Moreover, voluntary movements between two fixation points may be carried out at a frequency of about 2 per sec. The maximum time for fixation on each of these points during such a process must be less than $0.5 \mathrm{sec}$. The results given in Section III suggest that this period would not allow " accurate" fixation. Nevertheless with prolonged periods, the eye employs greater "accuracy of fixation", and a second process establishes the visual image on the central $20 \mathrm{~min}$. arc of the fovea.

The double process in establishing the visual image on the central region of the fovea after a transfer of the gaze might be explained on the basis of the structure of the retina. The information transmitted to the brain, which determines the size of the initial saccade, must be based on the distance between the central region and the point on the retina on which the eccentric image falls. But it is well known that as one proceeds outwards from the fovea along the retina the number of receptors associated with a single ganglion cell, and hence with a single nerve fibre, increases considerably. No definite 
information is available on the variation with eccentricity of the size of the independent receptor fields. Clemmesen (1945) suggests that the mean diameter of these fields increases up to an eccentricity of $3^{\circ}$ from the visual axis, but that outside this boundary the receptors are linked so diffusely that it is not possible to speak of separate functional units. Nevertheless, if the image of a target point falls on an eccentric part of the retina it is likely that a particular nerve fibre exhibits a maximum of activity. As the eccentricity increases it is probable that the region on which the image can fall and still produce maximum activity in the same nerve fibre also increases. The information transmitted to the brain with respect to the magnitude and direction of the movement necessary to bring the image on to the central region of the fovea cannot then be at all precise. This hypothesis explains qualitatively the nature of the movements found in the present investigation, although it does not explain the serious undershoots which have been obtained in some cases (Section IIA). More extensive data would be required to obtain a quantitative test. If the hypothesis is correct there should be a definite relation between the eccentricity of the fixation target exposed and the fixation " error" after completion of the saccadic movements. It is alsu possible that such an approach would provide information about the size of the receptor fields. During the second process by which the image is brought on to the central region, the involuntary flicks, which are a significant feature of eye movements which occur when the subject fixates (see e.g. Ditchburn and Ginsborg 1953), are of greater frequency, than during prolonged fixation. This phenomenon has been found by Barlow (1952), who suggests that, since the exposure of a new fixation target presents the subject with a stimulus of greater interest than is involved in prolonged fixation, the increased frequency of involuntary flicks enhances the performance of the retina. In fixation, during the periods between the flicks, the image is almost stationary relative to the retina. During these periods, therefore, it is possible that the receptors become fatigued. The consequent decrease in impulses passing down the optic nerve might act as a stimulus for a rotation of the eye to a new position and so cause a flick. Where the interest of the subject is low, as towards the end of prolonged fixation, other processes may be responsible for inhibiting the movements. In any case, it is now certain that movements of the visual image relative to the retina do play some part in visual perception since the contrast discrimination of the eye is considerably reduced when such movements are eliminated (Ditchburn and Ginsborg, 1952).

I wish to thank Dr. D. M. Maurice for his cooperation in acting as a subject, Mr. W. Martin for valuable technical assistance, and Professor R. W. Ditchburn for his constant guidance and advice. During the course of this work I was in receipt of a maintenance grant from the Department of Scientific and Industrial Research.

\section{REFERENCES}

Barlow, H. B. (1952). J. Physiol., Lond., 116, 290.

Clemmesen, V. (1944). Acta physiol. Scand., 9, suppl. 27.

Ditchburn, R. W. and Ginsborg, B. L. (1952). Nature, Lond., 170, 36.

-, L (1953). J. Physiol., Lond., 119, 1.

Ginsborg, B. L., and Heavens, O. S. (1951). Rev. sci. Instrum., 22, 114.

LORD, M. P., and Wright. W. D. (1949). Nature, Lond., 163, 803. 